\title{
Effect of repeated stress on novelty-induced antinociception in rats
}

I.L.S. Torres, A.P. Vasconcellos, S.N. Silveira Cucco and C. Dalmaz
Departamento de Bioquímica, Instituto de Ciências Básicas da Saúde, Universidade Federal do Rio Grande do Sul, Porto Alegre, RS, Brasil

\section{Correspondence \\ I.L.S. Torres \\ Departamento de Bioquímica \\ ICBS, UFRGS \\ Rua Ramiro Barcelos, 2600 \\ Anexo, Lab. 32 \\ 90035-003 Porto Alegre, RS \\ Brasil \\ Fax: +55-51-316-5540 \\ E-mail: torres@@zaz.com.br}

Research supported by PRONEX (No. 41.96.0904.00), CNPq and FAPERGS.

Received February 24, 2000 Accepted November 29, 2000

\section{Abstract}

There is extensive evidence that acute stress induces an analgesic response in rats. On the other hand, repeatedly stressed animals may present the opposite effect, i.e., hyperalgesia. Furthermore, exposure to novelty is known to induce antinociception. The effects of repeated restraint stress on nociception after exposure to novelty, as measured by the tail-flick latency (TFL), were studied in adult male rats. The animals were stressed by restraint $1 \mathrm{~h}$ daily, 5 days a week for 40 days. The control group was not submitted to restraint. Nociception was assessed with a tail-flick apparatus. After being familiarized with the TFL apparatus, each group was subdivided into two other groups, i.e., with or without novelty. Animals were subjected to the TFL measurement twice. For the animals exposed to novelty, the first TFL measurement was made immediately before, and the second 2 min after a 2min exposure to a new environment. While the control group presented an increased TFL after exposure to a novel environment, chronically stressed animals did not show this effect. These results suggest that repeated restraint stress induces an alteration in the nociceptive response, perhaps as a result of an alteration in endogenous opioids in these animals.

Individuals exposed to stressful conditions show an increase in pain threshold, known as stress-induced analgesia, which may present a different neurochemical basis related to stress severity $(1,2)$. Consistent with these findings, we have observed that acutely stressed rats (both males and females) show higher tail-flick latencies (TFL) when compared to unstressed controls. However, when the animals were previously submitted to repeated restraint they showed an adaptation which varied according to sex.
Chronically stressed females presented no effect compared to controls, suggesting habituation, i.e., they did not respond to the stress session with the characteristic analgesic effect. Male rats showed a decrease in TFL characterizing a hyperalgesic response both in the basal state and just after exposure to restraint (3).

Nociception has also been reported to be influenced by novelty (4-7). It has been shown that a 2-min exposure of rats to a novel environment is followed by mild antinoci- 
ception, as measured by the tail-flick method (6). The effect lasts 10 to $30 \mathrm{~min}$ and is not observed after a second exposure to the environment (6). Since chronically stressed animals present a hyperalgesic response (3), this raises the possibility of alterations induced by chronic stress in a variety of components associated with the regulation of nociception. We report here the effects of repeated restraint stress on novelty-induced antinociception, as measured by the TFL.

Male adult Wistar rats (60-70 days, mean weight $230 \mathrm{~g}$ at the beginning of treatment) from our breeding stock were used. The animals were housed in groups of 8 per cage and kept under a normal 12-h dark/light cycle with food and water ad libitum.

The animals were stressed by restraint 1 h daily, 5 days a week for 40 days (3). Restraint was applied by placing the animal in a $25 \times 7-\mathrm{cm}$ plastic bottle with a $1 \mathrm{~cm}$ hole at the far end for breathing. The animal was unable to move. The control group was not submitted to restraint. Pilot experiments have shown that handled animals present no difference in TFL compared to animals that were left undisturbed in their home cages. These procedures were performed always between 10:00 and 12:00 h. During the behavioral tests, that were carried out in the afternoon, the restraint sessions were maintained.

Nociception was assessed with a tailflick apparatus. Rats were wrapped in a towel and placed on the apparatus; the light source positioned below the tail was focused on a point $2.3 \mathrm{~cm}$ rostral to the tip of the tail. Deflection of the tail activated a photocell and automatically terminated the trial. Light intensity was adjusted so as to obtain a baseline TFL of 3 to $4 \mathrm{~s}$. A cut-off time of $10 \mathrm{~s}$ was used to prevent tissue damage.

The general procedure was as follows (5). On day 1, subjects were first familiarized with the TFL apparatus, the rats were then placed alone in a waiting cage for $2 \mathrm{~min}$, and then returned to their home cage. The waiting cage was similar to the home cages: a 65 × 25 × 25 -cm Plexiglas cage whose floor was covered with sawdust. On day 2 , each group was subdivided into two other groups, i.e., with or without novelty. Animals were subjected to the TFL measurement twice. For the animals exposed to novelty, the first TFL measurement was made immediately before, and the second 2 min after a 2-min exposure to a new environment (a $50 \mathrm{~cm}$ high, $40 \times 60 \mathrm{~cm}$ open field made of wood, with a linoleum floor and a front wall made of glass). This time was chosen because the effect of novelty on nociception is maximum at this time (6). Besides, it has been shown that the analgesic response to novelty is quite labile and susceptible to the influence of post-training stimuli from the environment (5). Between exposure to these situations and the second TFL measurement, the animals remained in the waiting cage. The second group (not exposed to novelty) was simply left in the waiting cage between the two TFL measurements.

Nociceptive data are expressed as percent maximum possible effect (\%MPE) according to the following formula (8): $\% \mathrm{MPE}=100 \mathrm{x}$ (postdrug latency - baseline latency)/(cut-off time - baseline latency).

Differences between the baseline latencies of control and repeatedly restrained rats were analyzed by the Student $t$-test. Twofactor analysis of variance (two-way ANOVA) was used to evaluate the effects of repeated stress treatment and exposure to novelty.

Results are shown in Figure 1. TFL is expressed as mean \%MPE \pm SEM for the repeated restraint and control groups measured in animals exposed or not to novelty. Two-way ANOVA showed an effect of exposure to novelty $(\mathrm{P}<0.01)$ and a significant interaction between chronic treatment and novelty $(\mathrm{P}<0.05)$. As can be observed, control rats presented an increased latency after being exposed to novelty, while chronically 
stressed rats showed no significant difference.

In this report we showed the effect of repeated restraint stress on nociception in rats exposed to novelty. It was observed that control rats presented novelty-induced antinociception, in agreement with previous literature reports, suggesting that certain forms of novelty might alter pain sensitivity (e.g., 5). On the other hand, when testing chronically stressed rats, we observed no significant difference between pre- and post-novelty latencies.

Repeated exposure to the same aversive event can lead to a process of adaptation to that stimulus. For example, rats may develop tolerance to stress-induced antinociception by repeated exposure to stress $(1,3,9)$. Different neurotransmitter systems have been suggested to play a role in this stress desensitization process (10-12), and interactions between stress and drugs that act on nociception have been studied in rats $(8,13,14)$. In addition, ACTH (adrenocorticotrophic hormone) and corticosteroids, which are released during stress situations, have been reported to have hyperalgesic properties $(15,16)$. In the present study, however, the reduced effect on nociception induced by novelty in treated rats was possibly not due to higher corticosterone levels, since when measured after exposure to restraint on the

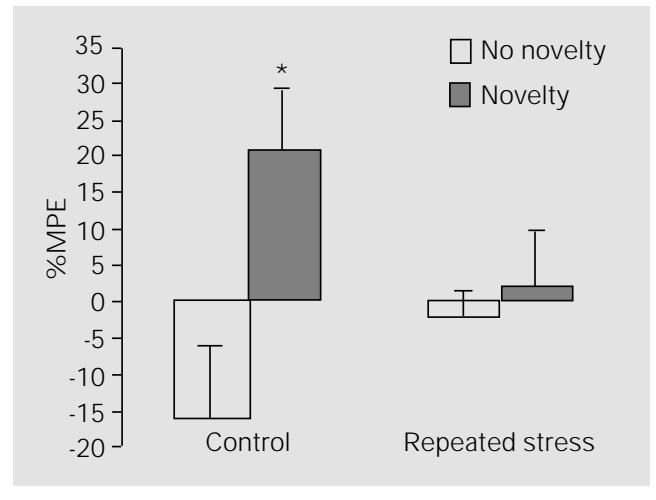

Figure 1 - Effect of exposure to a new environment on tail-flick latencies plotted as mean $( \pm$ SEM $)$ $\%$ maximum possible effect (\%MPE) ( $\mathrm{N}=8-10 \mathrm{rats} / \mathrm{group}$ ). Control and repeatedly restrained animals were subdivided into 2 other groups: with and without exposure to novelty, and tail-flick latencies were measured before (baseline) and after exposure to a novel environment. $* \mathrm{P}<0.05$ compared to animals not exposed to novelty (Student t-test test).

last day of treatment its levels were much lower than after a single restraint (data not shown). Moreover, several hours after the last restraint session, repeatedly stressed rats presented a nonsignificant decrease in plasma corticosterone when compared to controls (data not shown).

The present results point to an interaction between the antinociceptive effect of novelty and the adaptation induced by repeated stress. The precise mechanism involved in the development of this effect is not known, and further investigation of this phenomenon is required.

\section{Acknowledgments}

The authors gratefully acknowledge Dr. Maria Beatriz Ferreira for her kind assistance and advice.

\section{References}

1. Terman GW, Lewis J W \& Liebeskind J C (1986). Two opioid forms of stress analgesia: studies of tolerance and cross-tolerance. Brain Research, 368: 101-106.

2. Terman GW, Morgan MJ \& Liebeskind J C (1986). Opioid and non-opioid stress-analgesia from cold water swim: importance of stress severity. Brain Research, 372: 167-171.

3. Gamaro GD, Xavier MH, Denardin JD, Pilger J A, Ely DR, Ferreira MBC \& Dalmaz C (1998). The effects of acute and repeated restraint stress on the nociceptive response in rats. Physiology and Behavior, 63: 693-697.
4. Rochford J \& Stewart J (1987). Activation and expression of endogenous pain control mechanisms in rats given repeated nociceptive tests under the influence of naloxone. Behavioral Neuroscience, 101: 87-103.

5. Netto CA, Siegfried B \& Izquierdo I (1987). Analgesia induced by exposure to a novel environment in rats: effect of concurrent and post-training stressful stimulation. Behavioral and Neural Biology, 48: 304309.

6. Siegfried B, Netto CA \& Izquierdo I (1987). Exposure to novelty induces naltrexonereversible analgesia in rats. Behavioral
Neuroscience, 101: 436-438.

7. Rochford J , Dawes P \& Stewart J (1993). Novelty potentiation of novelty-induced hypoalgesia: characterization of the alphanoradrenergic receptor subtype. Pharmacology, Biochemistry and Behavior, 44: 381-386.

8. Calcagnetti DJ, Fleetwood SW \& Holtzman SG (1990). Pharmacological profile of the potentiation of opioid analgesia by restraint stress. Pharmacology, Biochemistry and Behavior, 37: 193-199.

9. Pavlovic Z\& Bodnar RJ (1993). Antinociceptive and hypothermic crosstolerance between continuous and intermittent 
cold-water swims in rats. Physiology and Behavior, 54: 1081-1084.

10. Cancela LM, Volosin M \& Molina VA (1990). Opioid involvement in the adaptive change of $5-\mathrm{HT}_{1 \mathrm{a}}$ receptors induced by chronic restraint. European J ournal of Pharmacology, 176: 313-319.

11. Girardot MN \& Holloway FA (1985). Chronic stress, aging and morphine analgesia: chronic stress affects the reactivity to morphine in young mature but not old rats. J ournal of Pharmacology and Experimental Therapeutics, 233: 545-553.
12. Girardot MN \& Holloway FA (1985). Effect of age and long-term stress experience on adaptation to stress analgesia in mature rats: role of opioids. Behavioral Neuroscience, 99: 411-422.

13. Calcagnetti DJ \& Holtzman SG (1991). Delta opioid antagonist, naltrindole, selectively blocks analgesia induced by DPDPE but not DAGO or morphine. Pharmacology, Biochemistry and Behavior, 38: 185190.

14. Appelbaum BD \& Holtzman SG (1985). Stress-induced changes in the analgesic and thermic effects of morphine administered centrally. Brain Research, 358: 303308.

15. Bertolini A, Poggioli R \& Ferrari W (1979). ACTH-induced hyperalgesia in rats. Experentia, 35: 1216-1217.

16. Capasso A, Di Giannuario A, Loizzo A, Pieretti S \& Sorrentino L (1992). Central interaction of dexamethasone and RU38486 on morphine antinociception in mice. Life Sciences, 51: 139-143. 